\title{
Management of hypertension in the elderly patient
}

This article was published in the following Dove Press journal:

Clinical Interventions in Aging

29 September 2009

Number of times this article has been viewed

\author{
Gordon Stewart Stokes 1,2 \\ 'Northern Clinical School, University \\ of Sydney, Sydney, New South Wales, \\ Australia; ${ }^{2}$ Department of Cardiology, \\ Royal North Shore Hospital, \\ St. Leonards, New South Wales, \\ Australia
}

\begin{abstract}
Hypertension in the elderly is associated with increased occurrence rates of sodium sensitivity, isolated systolic hypertension, and 'white coat effect'. Arterial stiffness and endothelial dysfunction also increase with age. These factors should be considered in selecting antihypertensive therapy. The prime objective of this therapy is to prevent stroke. The findings of controlled trials show that there should be no cut-off age for treatment. A holistic program for controlling cardiovascular risks should be fully discussed with the patient, including evaluation to exclude underlying causes of secondary hypertension, and implementation of lifestyle measures. The choice of antihypertensive drug therapy is influenced by concomitant disease and previous medication history, but will typically include a thiazide diuretic as the first-line agent; to this will be added an angiotensin inhibitor and/or a calcium channel blocker. Beta blockers are not generally recommended, in part because they do not combat the effects of increased arterial stiffness. The hypertension-hypotension syndrome requires case-specific management. Drug-resistant hypertension is important to differentiate from faulty compliance with medication. Patients resistant to third-line drug therapy may benefit from treatment with extended-release isosorbide mononitrate. A trial of spironolactone may also be worthwhile.
\end{abstract}

Keywords: hypertension, elderly, antihypertensive treatment, patient management

\section{Do the mechanisms underlying hypertension in the elderly differ from those in younger adults?}

Hypertension is a multifactorial disorder in which the mix of factors operative may vary according to age. Although there are no sharp dividing lines between age groups, age-associated trends can be recognized (Table 1). Recognition of these trends provides a basis for choosing age-specific approaches to clinical management.

Sodium sensitivity, defined as the extent of the rise in arterial blood pressure with an increase in sodium chloride intake, is heightened with age. ${ }^{1}$ In the elderly, limitation of dietary sodium intake and the use of diuretic agents show more effectiveness in controlling hypertension than in the young. ${ }^{2}$ The factors mediating the enhanced relationship between sodium status and change in blood pressure in the elderly are complex. They include an increased responsiveness of volume homeostasis to the level of salt intake, ${ }^{1}$ a salt-induced impairment of vascular nitric oxide (NO) production, and an increase in arterial stiffness. ${ }^{3}$ The systolic component of blood pressure is affected more than the diastolic., ${ }^{1,3}$

Isolated systolic hypertension (ISH) is characterized by systolic blood pressure $\geq 140 \mathrm{~mm} \mathrm{Hg}$ with diastolic blood pressure $<90 \mathrm{~mm} \mathrm{Hg}$, and (consequently) high pulse pressure.

\author{
Faculty of Medicine, University of Sydney, \\ PO Box 666, Mona Vale, NSW 2103 \\ Australia \\ Tel +6I 299992073 \\ Fax +6I 29997545 I \\ Email gstokes@med.usyd.edu.au
}

Correspondence: Gordon Stokes 
Table I Age-associated trends in clinical hypertension

I. Sodium sensitivity increases with age, as does the hypotensive response to diuretics

2. Isolated systolic hypertension becomes more frequent than systolic-diastolic hypertension

3. Arterial stiffness increases

4. There is a greater incidence of endothelial dysfunction

5. The frequency of 'white coat effect' increases

Among older subjects, ISH occurs more frequently than systolic-diastolic hypertension. In the US National Health and Nutrition Examination Survey III (1988-1991), ${ }^{4} 65 \%$ of subjects over the age of 60 were found to have ISH. By the sixth decade, ISH was found to be the overwhelmingly predominant form of uncontrolled hypertension, comprising $87 \%$ of the total. ISH arises more commonly from pre-existing normal blood pressure or high-normal blood pressure than from pre-existing diastolic hypertension. ${ }^{5}$ In patients originally treated for systolic-diastolic hypertension, later presentation with ISH may signify the onset of changes in the arterial tree which are not simply a consequence of the initial disorder. ${ }^{6}$

ISH is characterized by decreased arterial compliance, usually expressed as increase in arterial stiffness. There is controversy over the mechanism of the increased stiffness. Some attribute the effect to age-related loss of distensibility in the major central arteries ${ }^{7}$ as elastic tissue is progressively replaced with collagen. ${ }^{8}$ Other investigators attribute the increased arterial stiffness to endothelial dysfunction. ${ }^{9}$ Although both factors probably contribute, the effect of endothelial dysfunction is potentially the more correctable pharmacologically.

Endothelial dysfunction is caused by free oxygen radicals in the arterial wall and by the upstream effects of reduced distal vascular flow reserve. ${ }^{10}$ Accumulation of free oxygen radicals results from age-associated cardiovascular risk factors, including atherosclerosis, diabetes mellitus, and renal impairment as well as hypertension itself. ${ }^{10}$ Endothelial dysfunction manifests clinically as impairment in acetylcholine-stimulated, endothelium-dependent, NO-mediated vasodilatation: the depressor response to antihypertensive agents which operate through endotheliumdependent mechanisms is decreased, but the response to exogenous nitrates is preserved. ${ }^{11}$

The theory that attributes arterial stiffness to endothelial dysfunction is based on the following construct of how flow is propagated in the arterial system. ${ }^{12}$ The incident pressure wave generated by ventricular systole is transmitted freely along the viscoelastic aorta until it encounters arterial branching points and the peripheral resistance offered by muscular arterioles. Here a reflected wave is generated, returning retrograde towards the heart and augmenting the oncoming forward wave. The resulting pulse wave-form depends on a balance of endogenous vasoconstrictor and vasodilator factors in the arterial tree. When there is endothelial dysfunction, NO-mediated vasodilatation in the peripheral circulation is diminished. The loss of vasodilator potential results in increased amplitude and early return of the reflected wave, causing high pulse pressure. ${ }^{9}$ These characteristics are typical of the pulse wave found using applanation tonometry in groups of elderly patients with treatment-resistant ISH. ${ }^{11,13}$ The observation that treatment with long-acting nitrates potently decreased pulse wave reflection and lowered systolic blood pressure in these patients, despite their lack of response to other antihypertensive drugs, is consistent with the presence of endothelial dysfunction. ${ }^{11}$

The 'white coat effect' occurs when blood pressure is increased temporarily through an autonomic neural reaction triggered by the process of measurement. This effect increases with age. ${ }^{14}$ As the systolic component often rises more than the diastolic, the type of hypertension due to a 'white coat effect' could be mistaken for ISH. However, a 'white coat effect' can be confirmed by finding a major discrepancy between automated blood pressure values (in the normal range) and observer readings (high). ${ }^{14}$

\section{At what blood pressure level should antihypertensive medication be given and what is the treatment goal?}

In the year 2000, an advisory statement on systolic blood pressure in the elderly ${ }^{15}$ was issued to complement the Sixth Report of the Joint National Committee on the Prevention, Detection, Evaluation and Treatment of High Blood Pressure (JNC 6; 1997). The statement rejected the concept of age-adjusted blood pressure targets, adopting $140 / 90$ as the goal blood pressure (130/85 in diabetics) for elderly hypertensive subjects, including those with ISH. These guidelines were the same as those recommended by JNC 6 for adults in general, although in the case of ISH the evidence base was derived from two treatment trials (SHEP ${ }^{16}$ and SYST-EUR) ${ }^{17}$ in which the lower limit for subject entry was $160 / 90$. Since the time of publication of the advisory statement, further evidence from a large controlled trial 
(ALLHAT $)^{18}$ has supported the $140 \mathrm{~mm} \mathrm{Hg}$ goal for systolic blood pressure in ISH.

\section{To what age should we treat hypertension?}

Study of the treatment of hypertension in the elderly has a long history. In 1895, Professor Clifford Allbutt described a group of five patients aged between 65 and 80 years, whose high arterial pressure was relieved by treatment with mercurial diuretics and potassium iodide, inter alia, with apparent symptomatic benefit. ${ }^{19}$ Ambivalence about the value of treating hypertension in the elderly prevailed, however, for many decades after that time. In clinical practice, widening of the pulse pressure with age was seen as compensatory to 'hardening of the arteries,' so it was feared that lowering the arterial pressure could cause circulatory collapse; in particular, it was thought that the renal circulation in uremic patients might be vulnerable. ${ }^{20}$ As late as 1996-1997, $76 \%$ of a group of primary-care physicians in the USA stated that they would not initiate therapy for systolic blood pressure of $140-159 \mathrm{~mm} \mathrm{Hg}$ in patients over 70 years of age. ${ }^{21}$

It was not until controlled clinical trials were reported in 1985 and onwards, ${ }^{16,22-25}$ that the value of antihypertensive treatment in elderly patients started to become more universally accepted. In these trials, various combinations of diuretics, beta blockers, calcium channel blockers, and angiotensin-converting enzyme (ACE) inhibitors were given as the active therapy. By the year 2000, a meta-analysis of the trials completed in subjects aged 60 years or older, with systolic blood pressure $\geq 160 \mathrm{~mm} \mathrm{Hg}$ and diastolic blood pressure $\leq 90 \mathrm{~mm} \mathrm{Hg}$, had shown that active treatment reduced total mortality (by 13\%), and cardiovascular mortality (by 26\%), and stroke (by $30 \%$ ). ${ }^{26}$ This metaanalysis, together with a Cochrane review summarizing earlier data, ${ }^{27}$ helped clearly establish the value of antihypertensive agents for treating patients in their 7th and 8th decades. But questions remain about the benefit of such treatment in those even older.

Recently, the HYVET trial ${ }^{28}$ in 3,845 patients aged 80 years or older with systolic blood pressure $\geq 160 \mathrm{~mm} \mathrm{Hg}$ showed a $21 \%$ reduction in overall mortality and a $30 \%$ reduction in stroke after treatment for two years with a diuretic (indapamide sustained-release, $1.5 \mathrm{mg} /$ day) and, if required, an ACE inhibitor (perindopril 2-4 mg/day). It was notable in this trial that fewer adverse affects were reported with active treatment than with placebo. The results for HYVET differ from those of a meta-analysis (INDANA) published nine years earlier. ${ }^{29}$ In 1,670 patients aged
80 years or older, treated mainly with high-dose diuretics or beta-blockers, INDANA showed a 34\% reduction in stroke, but no benefit in overall mortality. The reason for the disparity between these reports may reside in the choice of drugs in INDANA: among the very elderly, high-dose diuretics may pre-dispose to electrolyte disorders and sudden death; beta-blockers are also less effective than ACE inhibitors in reducing blood pressure. ${ }^{30}$

Given that the benefit of stroke prevention for future lifestyle holds at any age, the clinical trial findings seem to indicate that there is virtually no age limit at which ageappropriate antihypertensive therapy should be withheld. However, a poor prognosis caused by concomitant systemic disease may influence a decision not to treat. Again, if multiple cardiovascular risk factors are present, the use of antihypertensive measures should be considered only in conjunction with the application of other cardiovascular-risk management strategies.

Before going on to describe drug therapy in the management of elderly hypertensive patients, it is necessary to consider the role of diagnostic screening and the application of non-drug therapeutic measures.

\section{An outline of steps in initial management}

The presence of systolic-diastolic hypertension, or ISH, should be confirmed by several office measurements of blood pressure, preferably at more than a single visit, and in the supine as well as the erect posture. Ideally, a 24-hour recording of ambulatory blood pressure is done to exclude 'white coat' effect and to determine the circadian pattern of blood pressure increase. The status of pre-existing medical disorders will need assessment, particularly those that increase cardiovascular risk, such as diabetes mellitus, hyperlipidemia, and chronic renal failure.

As part of the routine clinical examination of the patient, renal and endocrine causes of hypertension should be excluded. Investigation for sleep apnea may also be required. The tests recommended are included in Table 2. Plasma concentrations of electrolytes, uric acid, creatinine, renin, and aldosterone should be measured before starting drug therapy. Increased plasma creatinine concentration indicates renal impairment: further investigation may be needed to determine whether this finding is caused by renal artery constriction or outflow tract obstruction, both potentially remediable conditions. Hyperuricemia denotes a risk that gout may be precipitated if diuretic therapy is given. Hypokalemia and/or a raised renin-aldosterone ratio suggest primary 
Table 2 Initial investigations recommended for newly-presenting elderly hypertensive patients

Plasma electrolyte concentrations

Plasma creatinine concentration

Plasma uric acid concentration

Plasma renin activity

Plasma aldosterone concentration

Plasma lipids

Urine microscopy

24-hour urinary aldosterone excretion*

24-hour urinary catecholamine excretion*

Renal ultrasound

Computed tomography scan of renal arteries*

Overnight oximetry*

Echocardiography

Note: $*$ These tests are not needed in all patients.

aldosteronism, which can cause resistant hypertension; these findings would prompt a 24-hour collection of urine for determination of urinary aldosterone excretion rate, and further tests to detect a possible adrenal adenoma. Urinary catecholamines are measured if pheochromocytoma is to be excluded. Urinary microscopy and a renal ultrasound help in detection of underlying renal parenchymal disease or renal outflow obstruction. If an epigastric bruit is heard, or if there is disparity in renal size, computed tomography examination of the renal arteries may be required to exclude renal artery stenosis. If there is a history of daytime drowsiness or sleep disturbance, overnight oximetry should be done to exclude obstructive sleep apnea.

\section{Is diagnostic evaluation for underlying causes of hypertension worthwhile in the elderly?}

There is a tendency in clinical practice for diagnostic scrutiny in the elderly to be not as thorough as in younger hypertensive patients. It is sometimes argued that elderly subjects with underlying lesions causing secondary hypertension fare better with antihypertensive drug therapy than with surgical correction (achieved, for example, by renal angioplasty or adrenalectomy). In general, this is true for cases with renovascular hypertension, and possibly those with primary aldosteronism. However, when renal artery stenosis is causing renal impairment as well as hypertension, or if a unilateral adrenal adenoma is producing severe aldosteronism and treatment-resistant hypertension, surgery may be the better option. Additionally, knowledge of the diagnostic background is often important in selecting case-appropriate antihypertensive medication, such as spironolactone for primary aldosteronism caused by adrenal hyperplasia.

\section{To what extent can elderly patients with hypertension be involved in their own clinical management?}

The patient-doctor relationship is integral to a satisfactory outcome in managing any long-term medical disorder, and hypertension is no exception. The sequel to failed medical management of hypertension in the elderly may be a sudden stroke or heart attack, with severe disability or death. Yet, at the time of presentation most patients are suffering few or no symptoms. Part of the importance of the initial steps in detecting the presence of significant hypertension, and applying tests to exclude underlying causes, is the opportunity afforded to the doctor to establish the patient's trust and to lay the ground for later implementation of therapeutic interventions (which the patient may find onerous).

The interaction of hypertension and other risk factors, as well as the adverse effects of the therapy under consideration, should be explored with the patient. ${ }^{31}$ With the patient's permission, an accompanying spouse, relative or caregiver can be included in the discussion. Cardiovascular risk calculators based on algorithms relating multiple risk factors to outcomes such as heart attack, stroke and death may be used to help patients weigh up the advantages and disadvantages of risk modification programs. ${ }^{32}$ Therapeutic goals should be set by common agreement between patient and doctor, and appointments for follow-up visits planned. In addition to target levels of blood pressure, goals to be set might include, for example, a specified weight loss, a set restriction of dietary salt intake, or an increase in bodily exercise. Options in drug therapy should be discussed with the patient, ${ }^{31}$ and the importance of compliance with medication schedules should be stressed. In a small minority of patients, discussion of surgical intervention, such as adrenalectomy or repair of renal arteries will be required; foreseeably, renal sympathectomy might come to be considered in those subjects who are refractory to other measures..$^{33}$

\section{Are lifestyle modifications important in managing elderly hypertension?}

Body weight generally increases with age, and in many population studies, blood pressure levels have been shown to be directly related to body weight. Hypertension is more 
prevalent in overweight subjects and is often hard to control. ${ }^{34}$ In a recent study of 6,263 Spanish men and women, body mass and waist circumference showed a direct impact on the prevalence of hypertension and on the absence of control of blood pressure. ${ }^{35}$

The effect on blood pressure of weight loss through dieting varies from patient to patient. However, weight loss should be a goal considered in any elderly patient with a body mass index greater than 26 . Reduced alcohol intake is often the key factor in a successful outcome from dietary measures, resulting in impressive falls in both body weight and blood pressure.

Blood pressure has a direct relationship to alcohol consumption, independent of body weight. ${ }^{36}$ In many obese individuals, especially among men in developed societies, alcohol intake represents a potent calorific source. Thus, alcohol restriction can have a dual effect on blood pressure: that operating through weight reduction due to decreased intake of calories, and that due to prevention of the weightindependent pressor activity of alcohol.

Despite the evidence that sodium sensitivity is increased in the elderly, ${ }^{1}$ there has been a general lack of commitment by clinicians to the restriction of dietary sodium in elderly hypertensive patients. There are several reasons for this: the effects of sodium restriction on blood pressure in clinical trials have been variable; the extent of sodium deprivation required for an appreciable antihypertensive effect is uncertain; ${ }^{37}$ the need for dietary changes can be bypassed by inducing sodium loss with a diuretic. However, findings from controlled studies in older subjects with systolic hypertension have supported the use of low sodium diet (in the range 60-90 $\mathrm{mmol}$ per day) for decreasing blood pressure. ${ }^{38,39}$ In one study of 24 patients with ISH, a month of usual salt intake was compared to a month of reduced salt intake: a decrease in urinary sodium excretion from $175 \pm 51$ (standard deviation [SD]) to $87 \pm 38 \mathrm{mmol}$ per 24 hours was associated with a significant fall in systolic blood pressure from $166 \pm 19$ to $156 \pm 20 \mathrm{~mm} \mathrm{Hg} .{ }^{38}$ In another study of similar duration in 12 patients with less severe ISH, the corresponding urinary excretion values were respectively $135 \pm 14$ and $57 \pm 8$ (standard error [SE]) mmol per 24 hours; both the office and mean 24-hour ambulatory blood pressure values were significantly decreased in the low sodium phase. ${ }^{39}$

Considerable differences in sodium sensitivity are seen between individual hypertensive patients. ${ }^{1}$ A trial of low sodium diet may be worthwhile in elderly subjects with mild (Phase I) ISH: ${ }^{39}$ particular indications would include a high sodium excretion rate on usual diet, a willingness to modify salt intake, and a history of gout or diuretic-induced adverse events.

Regular aerobic exercise has been associated with modest blood pressure reduction in hypertensive patients overall. ${ }^{40}$ However, reports differ as to the effect of aerobic exercise on hypertension in the elderly. The efficacy of moderate physical exercise in controlling hypertension was investigated in a group of elderly women, nearly half of whom had ISH. Systolic blood pressure was found to be significantly lower among those moving more than five hours per day than among those moving less. ${ }^{41}$ However, another trial showed that arterial stiffness in ISH is not modified by moderate bicycle exercise. ${ }^{42}$ The efficacy of long-term exercise training in reversing or preventing arterial stiffness and hypertension needs further study. However, aerobic exercise increases plasma high-density lipoprotein cholesterol and lowers overall cardiovascular risk, and is of particular importance in hypertensive subjects with lipid disorders and diabetes mellitus. If arthritis limits walking or running in the elderly patient, wading or 'aquarobics' in a hydrotherapy pool are alternative ways to exercise.

\section{Which antihypertensive drug regimens are suitable for treating elderly patients?}

Selection of drug therapy for an individual patient is determined by considering the medical history, pre-existing therapy at baseline, and previous medication experiences.

The ALLHAT trial showed that a diuretic (chlorthalidone), given once-daily, was comparable in efficacy to a calcium channel blocker or ACE inhibitor for the treatment of hypertension, and slightly superior in preventing adverse cardiovascular outcomes. ${ }^{18}$ Evidence from a clinical study of diuretic use in ISH has been positive ${ }^{43}$ A diuretic, indapamide, was shown to be more selective than an angiotensin II receptor blocker or calcium channel blocker for decreasing systolic blood pressure and pulse pressure without lowering normal diastolic blood pressure levels ${ }^{43}$ as unduly low diastolic blood pressure is deemed to be deleterious in the elderly, this selectivity is a theoretical advantage.

Thus, a diuretic is a good first step in treating hypertension in the elderly, and a thiazide or thiazide-like diuretic is usually the most suitable to use. In elderly patients, some extra care is appropriate because of their sensitivity to diuretics. Inappropriately high doses may provoke hypotension, electrolyte disturbances or uremia. Hypokalemia is less likely to occur when a diuretic is combined with either a potassium conserving diuretic (spironolactone, amiloride) 
or an angiotensin inhibitor. Diuretics may be contraindicated in subjects with gout, hyperuricemia, diabetes, or renal impairment.

Alternatively, a calcium channel blocker, an ACE inhibitor or angiotensin II receptor blocker can be used at the first step. When considering the calcium channel blocker class, a dihydropyridine type of calcium channel blocker may be better suited than a nondihydropyridine type if the hypertension is severe or if there is coincident treatment with a negative inotrope (eg, a beta-blocker), while a nondihydropyridine may be preferred if there is a personal or family history of ischemic stroke. An ACE inhibitor or an angiotensin II receptor blocker may be given in preference to a calcium channel blocker as a first step when there are signs of heart failure or left ventricular hypertrophy. Centrally-acting sympathetic agonists (such as clonidine or guanfacine) have little role in the elderly, because of sedation and other side-effects. Use of peripheral alpha-adrenoceptor blockers is subject to a risk of postural hypotension. If beta blockers are part of pre-existing therapy, given, for example for arrhythmia or secondary prevention of myocardial infarction, then they should not be discontinued; however, they may show a limited effect on systolic blood pressure.

Many studies have shown that the effect of antihypertensive therapy can be enhanced by using combinations of agents from different classes. Hence, if one agent fails to achieve control of blood pressure, one of another class should be added and the dosages titrated for optimal effect. If needed, a third agent is used. Typically, triple therapy would include a diuretic, an ACE inhibitor or angiotensin II receptor blocker, and a calcium channel blocker. Failure to achieve the systolic blood pressure goal with three agents indicates a need for thorough clinical review to exclude any underlying cause that may have been unrecognized during initial evaluation of the patient.

\section{Why should beta blockers, prominent in treating hypertension over recent decades, be no longer favored for this condition?}

Beta blockers had a foundational role in the combined drug treatment of hypertension. They were used in combination with a diuretic for an active treatment arm in Systolic Hypertension in the Elderly Program (SHEP), ${ }^{16}$ and in other trials which showed a reduction of cardiovascular morbidity and mortality with combination therapy. However, in recent years their status in the treatment of ISH has become less assured. ${ }^{44} 46$
Findings from two major studies (LIFE ${ }^{47}$ and ASCOT-BPLA) ${ }^{44}$ indicated that beta blockers have less efficacy than other antihypertensive agents in preventing stroke. It has been suggested that beta blockers should be no longer used in the primary treatment of hypertension, ${ }^{48}$ especially not in the elderly. ${ }^{49}$ Also, in contradistinction to other key groups of antihypertensive agents, beta blockers do not decrease the arterial wave reflection often prominent in elderly hypertensive subjects, ${ }^{50,51}$ and do not rectify arterial stiffness. The Conduit Artery Function Evaluation study examined the chronic effects of atenolol \pm thiazide therapy versus amlodipine \pm perindopril therapy on central aortic blood pressures and hemodynamics. ${ }^{50}$ Despite similar reductions in brachial blood pressure with the two treatment arms, the central aortic pulse pressure was significantly less with amlodipine \pm perindopril, as was augmentation index (an indicator of arterial wave reflection). Also, central pulse pressure and pulse wave reflection were found to be significantly associated with a composite of cardiovascular and renal morbid events. Thus, agents which decrease wave reflection, such as amlodipine and perindopril, may not only lower central pressure more than agents that do not, such as the beta blockers, but also may be associated with a better clinical outcome. However, these negative findings about beta blockers do not contraindicate their use for secondary prevention in hypertensive subjects with a history of heart attack.

\section{Some management problems common in hypertension of the elderly 'White coat effect'}

As mentioned above, the condition of 'white coat hypertension' is more common in the elderly, and is often initially mistaken for ISH (see Table 3). In anticipation of a blood pressure measurement, the patient may become aware of this condition through a feeling of anxiety and an increased consciousness

Table 3 Management problems in elderly hypertensive patients

\begin{tabular}{ll}
\hline Problem & Steps indicated \\
\hline 'White coat effect' & Automated blood pressure recording \\
Hypertension-hypotension & Measure postural changes in blood \\
syndrome & pressure \\
Inadequate response to therapy & Check treatment compliance \\
& Perform pulse wave analysis \\
& Trial of long-acting nitrate \\
& Trial of spironolactone \\
\hline
\end{tabular}


of heart beat. Readings of blood pressure taken by an observer (physician or nurse) in the physician's office are high, while those taken at home by the patient are normal. The discrepancy may become more evident when the results from automated 24-hour ambulatory blood pressure recording are compared with office readings. It has been reported that the white coat response elicited by observer-conducted office blood pressure measurements can be virtually eliminated by recording blood pressure with an automated device while the patient rests alone in a quiet room. ${ }^{52}$ 'White coat hypertension' is deemed usually not to require treatment. However, a recent 10-year follow-up study of subjects with this syndrome showed that they may develop sustained hypertension, and may have a worse prognosis than normotensive subjects. ${ }^{53}$

\section{Hypertension-hypotension syndrome}

Elderly patients with orthostatic hypotension, a condition that can cause severe symptoms and morbidity, also may have supine hypertension. This combination has been termed the hypertension-hypotension syndrome. ${ }^{54}$ The hypotensive component reflects dysautonomia, for which various disorders may be responsible. These include spinal cord trauma or degeneration, autonomic neuropathy, neoplastic disease, and drugs. Low cardiac output or plasma volume depletion may contribute. The hypertensive component may reflect increased peripheral vascular resistance, caused by drugs used to control orthostatic symptoms or by vascular stiffness arising from long-standing cardiovascular disease.

The syndrome may escape detection if blood pressure is not checked in the supine as well as in the erect posture. Coexistence of supine hypertension and orthostatic hypotension poses a therapeutic dilemma, as treatment of one component of the syndrome can exacerbate the other. ${ }^{54}$ Use of tilt-table studies may be helpful in the diagnosis of the syndrome, and in the management of the symptoms. Specific diagnosis of the underlying cause is often not possible. Treatment of the syndrome requires careful titration of volume expanders, sympathomimetic agents or beta blockers, ${ }^{54}$ so as to control postural symptoms without increasing supine blood pressure.

\section{Inadequate response to therapy}

\section{Faulty compliance with therapy}

If the patient's blood pressure fails to respond to antihypertensive drug therapy, faulty compliance with medication should be excluded. When the patient is suffering from memory loss, the degree of compliance with medication may be difficult to ascertain. Observation by the dispensing pharmacist or the patient's carer may help. This is facilitated by asking the patient to use a multicompartment dispenser with clear plastic cover, through which each compartment holding tablets for a specified day of the week can be seen.

Having detected faulty compliance, an explanation should be sought. Is forgetfulness alone the cause? Does the patient understand the importance of taking the medication prescribed? Are there too many tablets, or is there difficulty in swallowing them? Can the patient afford the medication costs? Where possible, medication should be on a oncedaily basis, and the number of tablets to be taken should be minimized by the use of drug-combination tablets. Those readily available include a range of angiotensin inhibitors combined with a diuretic, and a calcium channel blocker combined with atorvastatin. Trials are in progress with the 'polypill', 55 which may eventually provide further simplification of drug therapy for multiple indications.

\section{Resistance to drug therapy}

Drug-resistant hypertension, common in ISH, can be said to exist when triple drug regimens fail to control the systolic blood pressure despite good compliance with therapy. Before concluding that true resistance is present, it is important to consider whether medications given to the patient for coexisting medical conditions are causing interference with antihypertensive treatment. In the elderly, the agents which most commonly cause this are non-steroidal antiinflammatory drugs, but sympathomimetics, some immunosuppressants (tacrilimus, cyclosporine), and steroid hormones may also be responsible. A careful drug history should be taken, and a trial of exclusion of any suspect compound may need consideration.

In true, treatment-refractory ISH, the pulse wave contor typically shows that the amplitude of the reflected wave is greatly increased. This effect is thought to be the outcome of stiffness of the conduit arteries. The mechanism producing this change is uncertain, but may relate to endothelial dysfunction and a consequent deficiency in NO-mediated vasodilator activity.

\section{Management of drug-resistant hypertension}

The use of exogenous NO donors to decrease wave reflection and pulse pressure

Theoretical basis

The unique ability of NO donors to rapidly decrease pulse wave reflection has been known for many years. ${ }^{56}$ This property is due to direct effects of NO on peripheral 
muscular arteries, ${ }^{57}$ and results in a fall of pulse pressure and systolic blood pressure with little change in diastolic pressure. These effects are most striking in elderly patients with ISH, in whom the amplitude of wave reflection reduction may be decreased by $40 \%$, or more, by treatment with $\mathrm{NO}$ donors such as isosorbide mononitrate (ISMN). ${ }^{58}$ Diuretics, calcium channel blockers and angiotensin II inhibitors also can decrease wave reflection, although not to the same extent nor as rapidly as NO donors. ${ }^{58}$ The unique adjuvant effect of NO donors results from their direct action on the cyclic guanosine monophosphate (GMP) in vascular smooth muscle, producing vasorelaxation through activation of cyclic GMP-dependent protein kinase. This action is endothelium-independent and is different from the vasodilator effects of angiotensin II inhibitors and calcium channel blockers, which are subject to modulation by endogenous NO release in the vasculature. Angiotensin II inhibitors are known to increase NO bioavailability, ${ }^{59}$ and to improve endothelium-dependent vasodilatation with longterm use ${ }^{60,61}$ but not with short-term use. ${ }^{62}$ It may take three years for ACE inhibitors to restore impaired acetylcholineresponsive vasorelaxation in hypertension. ${ }^{63}$ Thus, failure of response to conventional antihypertensive drugs such as angiotensin II inhibitors may be due, at least in the short term, to endothelial dysfunction. It has been speculated that NO donors, by maintaining peripheral vasodilatation through an endothelium-independent vasodilator action until endothelial recovery occurs, could have their greatest adjunctive effect in situations where endothelial dysfunction is currently limiting the response to other agents. ${ }^{11}$

\section{Indications for a trial of ISMN}

Conditions suggesting that an extended-release nitrate may be effective for the treatment of ISH are, first, failure of standard regimens of combination antihypertensive therapy to decrease systolic blood pressure below $150 \mathrm{~mm} \mathrm{Hg}$, and, second, a pulse pressure greater than $60 \mathrm{~mm} \mathrm{Hg}$. If a pulse wave analyzer, such as the SphygmoCor applanation tonometer (AtCor Medical, West Ryde, New South Wales, Australia), is available, the presence of a large reflection component in the pulse wave profile is a further indication for the use of a nitrate.

\section{Implementing nitrate therapy}

The nitrate recommended for treatment of ISH is extendedrelease ISMN, in a dose of 60-120 mg/day. This should be given as an adjunct to the standard antihypertensive drugs with which the patient is being currently treated. ISMN should be given once-daily, thus allowing for a 12-hour interval when plasma nitrate level is low between doses: this may prevent the development of nitrate tolerance. A starting dose of 30-60 mg (as an ISMN extended-release formulation) is recommended, to be given once-daily in the morning. This may be increased rapidly to $120 \mathrm{mg}$ once-daily if required to reach target systolic blood pressure. If the patient has a history of headaches, the starting dose should be $30 \mathrm{mg}$, in conjunction with paracetamol, $1 \mathrm{~g}$, per mouth. If headache occurs, it often abates after the first few doses. The dosage can usually be increased to $120 \mathrm{mg}$ daily without headache re-appearing. Apart from headache, which is reported in about $10 \%$ of patients, side-effects are rare. An important contra-indication to the introduction of nitrates in this, as in other clinical settings, is the concurrent use of sildenafil for erectile impotence.

\section{Timing of dosage in combined use of nitrates}

\section{with other agents}

Extended-release ISMN should be given in the early morning, at about $8 \mathrm{am}$, as a single daily dose. This allows the effect to operate mainly during daylight hours when blood pressure is usually highest. Previous therapy should be continued without dosage changes, but the timing of administration may need adjustment. Consideration should be given to administering the daily dose of extended-release forms of calcium channel blocker or angiotensin II inhibitor drugs at night, so that their peak levels will occur in the early morning. This should keep blood pressure low at a time when circadian cardiovascular risk is high, and peak blood levels of ISMN are yet to be reached after the morning dose.

\section{The use of aldosterone inhibitors}

in resistant hypertension

Reduction of blood pressure by aldactone has been shown in many clinical studies over the last 43 years (cited by Chapman and colleagues). ${ }^{64}$ Some of the patient populations studied had 'low renin hypertension' or raised aldosterone to renin ratios, indicating the possibility that cases of primary aldosteronism may have been included. In a patient group from which such cases were excluded, aldactone still showed antihypertensive activity when used as an add-on medication. ${ }^{65}$ As spironolactones (aldactone and eplerenone) can precipitate hyperkalemia (which may a particular hazard in elderly people with renal impairment), this class of diuretic is not recommended as first-line treatment in the elderly. However, aldactone may be an effective adjunct for treating resistant hypertension in elderly patients with intact renal function. The starting dose of aldactone for treating elderly 
subjects with resistant hypertension should be $12.5-25 \mathrm{mg}$ per day. The dosage can be increased to $50-75 \mathrm{mg}$ per day, according to clinical response. The rate of onset of response is slow. Dosage increments should not be made more frequently than every two weeks. Serum potassium concentration should be checked during dose titration, especially in patients who are already receiving an ACE inhibitor or angiotensin II receptor antagonist. ${ }^{65}$ Gynecomastia may occur in males receiving long-term therapy with aldactone.

In addition to resistant cases with and without known primary hyperaldosteronism, those with obstructive sleep disorder (OSD) have come also under consideration for possible aldactone therapy. OSD is common amongst patients with resistant hypertension, ${ }^{66}$ and increasing severity of OSD is associated with worse control of blood pressure. ${ }^{67}$ Possible causal links between OSD and hypertension include aldosterone excess. ${ }^{68}$ If patients with OSD and hypertension should fail to tolerate continuous positive airways pressure (CPAP) therapy, then it seems plausible that aldactone might be useful for lowering their blood pressure: however, this proposition has not yet been tested in clinical trials.

\section{Conclusions}

Principles in the treatment of hypertension in the elderly have much in common with those applying to younger age groups. In particular, there appears to be no cogent reason to withhold active therapy for hypertension in persons in their 8 th or 9th decades of life, nor to modify the blood pressure thresholds above which treatment is recommended. Also, the need for careful investigation and treatment for underlying causes of secondary hypertension still applies in older persons. Issues especially requiring prompt separation from mainstream management in the elderly are 'white coat hypertension' and hypertension-hypotension syndrome. Sensitivity of the elderly to sodium and to diuretics enhances rather than reduces the value of a thiazide diuretic as the first-line choice in mainstream treatment. The second-line option is either an angiotensin inhibitor (ACE inhibitor/angiotensin II receptor blocker) or a calcium antagonist, depending upon the clinical setting. ISH, the prevalent form of hypertension in the elderly and characterized by arterial stiffness, is often resistant to combination therapy with a thiazide diuretic, angiotensin inhibitor, and calcium antagonist. The deficiency of endothelial NO-dependent vasodilatation that contributes to arterial stiffness may be bypassed by adjunctive treatment with isosorbide mononitrate. An alternative strategy for bringing resistant ISH under control is to add aldactone, a spironolactone diuretic, to existing therapy.

\section{Disclosure}

The author reports no conflicts of interest in this work.

\section{References}

1. Weinberger MH, Miller JZ, Luft FC, Grim CE, Fineberg NS. Definitions and characteristics of sodium sensitivity and blood pressure resistance. Hypertension. 1986;8:127-134.

2. De Wardener HE, MacGregor GA. Sodium and blood pressure. Curr Opin Cardiol. 2002;17:360-367.

3. Bagrov AY, Lakatta EG. The dietary sodium-blood pressure plot "stiffens". Hypertension. 2004;44:22-24.

4. Franklin SS, Jacobs MJ, Wong ND, L'Italien GJ, Lapuerta P. Predominance of isolated systolic hypertension among middle-aged and elderly US hypertensives. Analysis based on National Health and Nutrition Examination Survey (NHANES) III. Hypertension. 2001;37: 869-874.

5. Franklin SS, Pio JR, Wong ND, et al. Predictors of new-onset diastolic and systolic hypertension. The Framingham Heart Study. Circulation. 2005;111:1121-1127.

6. Franklin SS. Arterial stiffness and hypertension. A two-way street? Hypertension. 2005;45:349-351.

7. Mitchell GF, Lacourciere Y, Oellet JP, et al. Determinants of elevated blood pressure in middle-aged and older subjects with uncomplicated systolic hypertension: the role of proximal aortic diameter and the aortic pressure-flow relationship. Circulation. 2003;108:1592-1598.

8. Virmani R, Avolio AP, Mergner WJ, et al. Effect of aging on aortic morphology in populations with high and low prevalence of hypertension and atherosclerosis. Am J Pathol. 1991;139:1119-1129.

9. O'Rourke, Nichols WW. Aortic diameter, aortic stiffness, and wave reflection increase with age and isolated systolic hypertension. Hypertension. 2005;45(part 2):652-658.

10. Kojda G, Harrison DG. Interactions between NO and reactive oxygen species: pathophysiological importance in atherosclerosis, hypertension, diabetes and heart failure. Cardiovasc Res. 1999,43:562-571.

11. Stokes GS. Nitrates as adjunct hypertensive treatment. Curr Hypertens Rep. 2006;8:60-68.

12. Vasan SV. Pathogenesis of elevated peripheral pulse pressure: some reflections and thinking forward. Hypertension. 2008;51:33-36.

13. Stokes GS, Ryan M. Can extended-release isosorbide mononitrate be used as adjunctive therapy for systolic hypertension? An open study employing pulse-wave analysis to determine effects of antihypertensive therapy. Am J Geriat Cardiol. 1997;6:11-19.

14. Amado P, Vasconcelos N, Santos I, et al. Arterial hypertension difficult to control in the elderly patient. The significance of the "white coat effect”. Rev Port Cardiol. 1999;18:897-906.

15. Izzo JL, Levy D, Black HR. Importance of systolic blood pressure in older Americans. Hypertension. 2000;35:1021-1024.

16. SHEP Cooperative Research Group. Prevention of stroke by antihypertensive drug treatment in older persons with isolated systolic hypertension: final results of the Systolic Hypertension in the Elderly Program (SHEP). JAMA. 1991;265:3255-3264.

17. Staessen JA, Thijs L, Fagard R, et al. Predicting cardiovascular risk using conventional vs ambulatory blood pressure in older patients with systolic hypertension: Systolic Hypertension in Europe Trial Investigators. JAMA. 1999;282:539-546.

18. The ALLHAT Officers and Coordinators for the ALLHAT Collaborative Research Group. Major outcomes in high-risk hypertensive patients randomized to angiotensin-converting enzyme inhibitor or calcium channel blocker vs diuretic. The antihypertensive and lipidlowering treatment to prevent heart attack trial (ALLHAT). JAMA. 2002;288:2981-2997.

19. Allbutt C. Senile plethora or high arterial pressure in elderly persons. Lectures to the Hunterian Society of London. 1895, February 27th, lecture 1. Cited in Swales JD, editor. Classic Papers in Hypertension: Blood pressure and renin. London, UK: Science Press; 1987. p. 104-123. 
20. De Wardener H. The Kidney: An outline of abnormal structure and function. London, UK: Churchill; 1961.

21. Hyman DJ, Pavlik VN. Self-reported hypertension treatment practices among primary care physicians: blood pressure thresholds, drug choices, and the role of guidelines and evidence-based medicine. Arch Intern Med. 2001;160:2281-2286.

22. Amery A, Birkenhager W, Brixko P, et al. Mortality and morbidity results from the European Working Party on High Blood Pressure in the Elderly trial. Lancet. 1985;1:1349-1354.

23. Dahlof B, Lindholm LH, Hansson L, et al. Morbidity and mortality in the Swedish Trial in Old Patients with Hypertension (Stop-Hypertension). Lancet. 1991;338:1281-1285.

24. Staessen JA, Fagard R, Thijs L, et al. Randomised double-blind comparison of placebo and active treatment for older patients with isolated systolic hypertension. Lancet.1997;350:757-764.

25. Reid CM, Ryan P, Wing LMH. The 2nd Australian National Blood Pressure Study (ANBP2). In: Black HR, editor. Clinical trials in Hypertension. New York, NY: Marcel Dekker; 2001. p. 587-604.

26. Staessen JA, Gasowski J, Wang JG, et al. Risks of untreated and treated isolated systolic hypertension in the elderly: meta-analysis of outcome trials. Lancet. 2000;355(9207):865-872.

27. Mulrow CD, Lau J, Cornell J, Brand M. Pharmacotherapy for hypertension in the elderly. Cochrane Database Syst Rev 1998;2:CD000028.

28. Beckett NS, Peters R, Fletcher AE, et al. for the HYVET Study Group. Treatment of hypertension in patients 80 years of age or older. $N$ Engl J Med. 2008;359:971-974.

29. Gueyffier F, Bulpitt C, Boissel JP, et al. Antihypertensive drugs in very old people; a subgroup meta-analysis of randomised controlled trials. Lancet. 1999;353(9155):793-796.

30. Dahlof B, Sever PS, Poulter NR, et al. Prevention of cardiovascular events with an antihypertensive regimen of amlodipine adding perindopril as required versus atenolol adding bendroflumethiazide as required, in the Anglo-Scandinavian Cardiac Outcomes Trial-Blood Pressure Lowering Arm (ASCOT-BPLA): a multicentre randomised controlled trial. Lancet. 2005;366:895-906.

31. Foody JM, Chaudry SI, Krumholz HM. Systolic hypertension in older persons: complexities in clinical decision making. Am J Geriat Cardiol 2005;14(6):325-330.

32. Hippiley-Cox J, Coupland C, Vinogradova Y, Robson J, May M, Brindle P. Derivation and validation of QRISK, a new cardiovascular disease risk score for the United Kingdom: prospective open cohort study. BMJ. 2007;335:136.

33. Krum H, Schlaich M, Whitbourn R, et al. Catheter-based renal sympathetic denervation for resistant hypertension: a multicentre safety and proof-of-principle cohort study. Lancet. 2009;373;1275-1281.

34. Bramlage P, Pittrow D, Wittchen HU, et al. Hypertension in overweight and obese primary care patients is highly prevalent and poorly controlled. Am J Hypertens. 2004;17(10):904-910.

35. Redon J, Cea-Calvo 1, Mareno B, et al. Independent impact of obesity and fat distribution in hypertension prevalence and control in the elderly. J Hypertens. 2006;26:1757-1764.

36. Cooke KM, Frost GW, Thornell IR, Stokes GS. Alcohol consumption and blood pressure: survey of the relationship at a health screening clinic. Med J Aust. 1982;1:65-69.

37. Townsend MS, Fulgoni VL III, Stern JS, et al. Low mineral intake is associated with high systolic blood pressure in the Third and Fourth National Health and Nutrition Examination Surveys. Could we all be right? Am J Hypertens. 2005;18:261-269.

38. He FJ, Markandu ND, MacGregor GA. Modest salt reduction lowers blood pressure in isolated systolic hypertension and combined hypertension. Hypertension. 2005;46:66-70.

39. Gates PE, Tanaka H, Hiatt WR, Seals DR. Dietary sodium restriction rapidly improves large elastic artery compliance in older adults with systolic hypertension. Hypertension. 2004;44:35-41.

40. Whelton SP, Chin A, Xin X, He J. Effect of aerobic exercise on blood pressure: a meta-analysis of randomized, controlled trials. Ann Intern Med. 2002;136:493-503.
41. Brennan P, Pescatello LS, Bohannon RW, et al. Time spent moving is related to systolic blood pressure among older women. Prev Cardiol. 2005;8:160-164.

42. Ferrier KE, Waddell TK, Gatzka CD, et al. Aerobic exercise training does not modify large-artery compliance in isolated systolic hypertension. Hypertension. 2001;38:222-226.

43. London G, Schmieder R, Calvo C, Asmar R. Indapamide SR versus candesartan and amlodipine in hypertension: the X-CELLENT Study. Am J Hypertens. 2006;19:113-121.

44. Dahlof B, Sever PS, Poulter NR, et al. Prevention of cardiovascular events with an antihypertensive regimen of amlodipine adding perindopril as required versus atenolol adding bendroflumethiazide as required, in the Anglo-Scandinavian Cardiac Outcomes Trial-Blood Pressure Lowering Arm (ASCOT-BPLA): a multicentre randomised controlled trial. Lancet. 2005;366:895-906.

45. Messerli FH, Grossman E, Goldbourt U. Are beta-blockers efficacious as first-line therapy for hypertension in the elderly? A systematic review. JAMA. 1998;279:1903-1907.

46. Carlberg B, Samuelsson O, Lindholm LH. Atenolol in hypertension: Is it a wise choice? Lancet. 2004;364:1684-1689.

47. Kjeldsen SE, Lyle PA, Kizer JR, et al. The effects of losartan compared to atenolol on stroke in patients with isolate systolic hypertension and left ventricular hypertrophy. The LIFE study. J Clin Hypertens. 2005;7:152-158.

48. Lindholm LH, Carlberg B, Samuelsson O. Should beta blockers remain first choice in the treatment of primary hypertension? A meta-analysis. Lancet. 2005;366:1545-1553.

49. Khan N, McAlister FA. Re-examining the efficacy of $\beta$-blockers for the treatment of hypertension: a meta-analysis. CMAJ. 2006;174:1737-1742.

50. Williams B, Lacy PS, Thom SM, et al. The CAFE Investigators, for the Anglo-Scandinavian Cardiac Outcomes Trial (ASCOT) Investigators, CAFE Steering Committee and Writing Committee. Differential impact of blood pressure-lowering drugs on central aortic pressure and clinical outcomes: principal results of the conduit artery function evaluation (CAFE) study. Circulation. 2006;113:1213-1225.

51. Mckenzie IS, McEniery CM, Dhakam Z, Brown MJ, Cockcroft JR, Wilkinson IB. Comparison of the effects of antihypertensive agents on central blood pressure and arterial stiffness in isolated systolic hypertension. Hypertension. 2009;54:409-413.

52. Myers MG, Valdivieso M, Kiss A. Use of automated office blood pressure measurement to reduce the white coat response. J Hypertens. 2009;27:280-286.

53. Mancia G, Bombelli M, Faccheti R, et al. Long-term risk of sustained hypertension in white coat or masked hypertension. Hypertension. 2009;54:226-232.

54. Naschitz JW, Slobodin G, Elias N, Rosner I. The patient with supine hypertension and orthostatic hypotension: a clinical dilemma. Postgrad Med J. 2006;82:246-253.

55. Combination Pharmacotherapy and Public Health Research Working Group. Combination pharmacotherapy for cardiovascular disease. Ann Intern Med. 2005;143:593-599.

56. Murrell W. Nitroglycerine as a remedy for angina pectoris. Lancet. 1879;80:80-227.

57. Pauca AL, Kon ND, O'Rourke MF. Benefit of nitroglycerin on arterial stiffness is directly due to effects on peripheral arteries. Heart. 2005;91:1428-1432.

58. Stokes GS, Barin ES, Gilfillan KL. Effects of isosorbide mononitrate and AII inhibition on pulse wave reflection in hypertension. Hypertension. 2003;41:297-301.

59. Brosnan MJ, Hamilton CA, Graham D, et al. Irbesartan lowers superoxide levels and increases nitric oxide bioavailability in blood vessels from spontaneously hypertensive stroke-prone rats. J Hypertens. 2002;20:281-286

60. Schiffrin EL, Deng Li Y. Comparison of effects of angiotensin I-converting enzyme inhibition and [beta]-blockade for 2 years on function of small arteries from hypertensive patients. Hypertension. 1995; 25(part 2):699-670 
61. Ghiadoni L, Magagna A, Versari D, et al. Different effect of antihypertensive drugs on conduit artery endothelial function. Hypertension. 2003;41:1281-1286.

62. Kiowski W, Linder L, Nuesch R, et al. Effect of cilazopril on vascular structure and function in essential hypertension. Hypertension. 1996;27(part 2):371-376.

63. Rizzoni D, Muiesan ML, Porteri E, et al. Effects of long-term antihypertensive treatment with lisinopril on resistance arteries in hypertensive patients with left ventricular hypertrophy. J Hypertens. 1997;15:197-204.

64. Chapman N, Dobson J, Dobson S, et al. Effect of spironolactone on blood pressure in subjects with resistant hypertension. Hypertension. 2007;49:839-845.
65. Zannad F, Lane DA, Shah S, Beevers GD. Low-dose spironolactone in the management of resistant hypertension: a surveillance study. J Hypertens. 2007;25:747-750.

66. Logan AG, Perlikowski SM, Mente A, et al. High prevalence of unrecognized sleep apnea: implications for cardiac and vascular disease. JAMA. 2001;19:2271-2277.

67. Grote L, Hedner J, Peter JH. Sleep-related breathing disorder is an independent risk factor for uncontrolled hypertension. J Hypertens. 2000;18:679-685.

68. Calhoun DA, Nishizaka MK, Zaman MA, Harding SM. Aldosterone excretion among subjects with resistant hypertension and symptoms of sleep apnea. Chest. 2004;125:112-117.
Clinical Interventions in Aging

\section{Publish your work in this journal}

Clinical Interventions in Aging is an international, peer-reviewed journal focusing on evidence-based reports on the value or lack thereof of treatments intended to prevent or delay the onset of maladaptive correlates of aging in human beings. This journal is indexed on PubMed Central, MedLine, the American Chemical Society's 'Chemical

\section{Dovepress}

Abstracts Service' (CAS), Scopus and the Elsevier Bibliographic databases. The manuscript management system is completely online and includes a very quick and fair peer-review system, which is all easy to use. Visit http://www.dovepress.com/testimonials.php to read real quotes from published authors.

Submit your manuscript here: http://www.dovepress.com/clinical-interventions-in-aging-journal 